\title{
Influence of freeze-drying conditions on orange powder flowability
}

\author{
Uscanga, $\mathbf{M}^{\mathrm{ab}}$;; Silva, A $^{\mathrm{a}}$; Egas, L..$^{\mathrm{a}}$; Camacho, M.M. ${ }^{\mathbf{b}}$; Martínez-Navarrete, $\mathbf{N}^{\mathrm{b}}$ \\ ${ }^{a}$ Universitat Politècnica de València, Valencia, Spain \\ b Instituto Tecnológico de Veracruz, Veracruz, México.
}

*E-mail of the corresponding author: mdmcamvi@tal.upv.es

\begin{abstract}
Freeze-drying may be a good alternative to get less perishable fruit products. The objective of the present study was to evaluate the impact of freeze-drying conditions of an orange puree on some flow related and rehydration properties of the obtained powders. The results showed that the application of heat during freeze-drying does not affect the parameters studied. However, the partial dehydration of the initial sample results in a lesser porosity and wettability values.
\end{abstract}

Keywords: freeze-drying; porosity; wettability; angle of repose; particle size distribution. 


\section{Introduction}

Fruits are basic foods and of great interest in human nutrition. The benefit of fruit consumption in health seems to be related to the presence of various compounds that belong to the group of phytochemical or bioactive substances. The daily consumption of fruits, in sufficient quantity and in a well-balanced diet, helps to avoid serious diseases, such as heart disease, cardiovascular accidents, diabetes and cancer, as well as deficiencies of important micronutrients and vitamins ${ }^{[1]}$. However, the consumption of fruit has declined gradually in recent years, since they have changed the eating habits of society. Despite the great benefits of fruit consumption, two major problems limit its availability, its seasonality and its short shelf life.

The orange is the fruit of the sweet orange tree that belongs to the genus Citrus of the Rosacea's family. Its nutritional composition highlights its low energy value, thanks to its high water content and its high content of vitamin $\mathrm{C}$, folic acid and minerals such as potassium, magnesium and calcium. It contains appreciable amounts of beta-carotene, responsible for its typical color and known for its antioxidant properties, in addition to malic, oxalic, tartaric and citric acids, which enhance the action of vitamin $\mathrm{C}$. The amount of fiber is appreciable and this is found mainly in the white part between the pulp and the bark, so that its consumption favors the intestinal transit. The orange has been selected as the object of this study because it is one of the most typical products of the country. Due to the great production that exists, Spain has become one of the largest producers of orange in the world and an asset for export. For this reason the orange represents a product of great importance in the economy of the country.

Currently, new alternatives for the development of less perishable products are being proposed. Powdered products represent a good option as healthy foods, lengthening the useful life of the product and facilitating its transport. Freeze-drying is a technology that consists of dehydrating the freeze product at low pressure. The process does not require the application of high temperatures, however its high cost has limited its use in the food industry. Therefore, the main interest is to find alternatives to minimize energy consumption, in order to decrease the process cost preserving the quality of the product. In this study, two options are proposed for this purpose, as they are the partial dehydration of the feed food and the application of heat during the process. However, this may affect the characteristics of the obtained powder in terms of the size and density of the particles, their porosity and surface properties, among others. In turn, these characteristics will determine the characteristic flow and / or rehydration behavior of a powder. Taking into account all of the above, the objective of this work was to know the impact of a pre-dehydration treatment and of the application of shelf temperature while the freeze-drying of a formulated orange puree on the properties of the obtained powder. 


\section{Materials and Methods}

\subsection{Raw material and formulation}

Orange (Citrus $x$ sinensis var. Navelina) always acquired in the same chain of supermarkets in Valencia was used for the study. To obtain the orange puree, the fruit was washed, peeled, cut and crushed in a food processor (Thermomix TM 21, Vorwek, Spain). Gum Arabic and bamboo fiber (5 and $1 \mathrm{~g}$, Shaurlau-Vitacel, respectively) were added to $100 \mathrm{~g}$ puree and homogenized with the same food processor. The ${ }^{\circ}$ Brix of the mixture were measured (Refractometer Mettler Toledo 30Px), this being $17^{\circ} \mathrm{B}$. A part of this orange formulation, named $\mathrm{O}$, was reserved. The rest of the sample was partially dehydrated (Moulinex Ultimys Duocombi, 600W) by heating in short period times until the sample reached $22{ }^{\circ} \mathrm{B}$. This partially dehydrated orange formulation was named OD.

\subsection{Freeze-drying}

Each formulation was placed in aluminium plates, $1 \mathrm{~cm}$ thickness, and freeze in a LIEBHERR MEDLINE equipment at $-45^{\circ} \mathrm{C}$ for 48 hours. The freeze-drying step was carried out in a Telstar Lyo Quest 55 equipment operating at $-50{ }^{\circ} \mathrm{C}$ in the condenser and at 0.063 mbar, for 48 hours. Samples O and OD were freeze-dried with the shelfs at room temperature and sample $\mathrm{O}$ was also freeze-dried at $40{ }^{\circ} \mathrm{C}$ in the shelf (sample O40).

\subsection{Obtaining powder}

The cakes obtained from the freeze dryer were crushed in a food processor (Thermomix TM 21, Vorwek, Spain), at speed 5 for $20 \mathrm{~s}$ to obtain a powder. Batches of about $40 \mathrm{~g}$ of the powder were sieved through $800 \mu \mathrm{m}$ mesh, with the corresponding top and bottom placed, to select the particles lower than this size which were characterized in the properties described below. A vibrating drum (AMP0.40, CISA, Barcelona, Spain), $50 \mathrm{~Hz}$, for 5 minutes was used to this end.

\subsection{Particle size distribution}

A batch of about $40 \mathrm{~g}$ of the obtained powder lower than $800 \mu \mathrm{m}$ was again sieved in the same vibrating drum and conditions. The mesh of the sieves used in this case were 500, 300, 200, 150 and $100 \mu \mathrm{m}$, with a top and a botton placed in the column of sieves. The powder collected in each sieve and in the bottom was weighed.

\subsection{Angle of repose}

This is the angle formed between the slope of the product when dropped down from $5 \mathrm{~cm}$ height from the horizontal surface. The test consists of pouring $15 \mathrm{~g}$ of powdered product in a funnel (top diameter $=80 \mathrm{~mm}$, stem $=11 \mathrm{~mm}$ diameter, $29 \mathrm{~mm}$ length; Overall height $=85$ 
$\mathrm{mm}$ ) and measuring the diameter and height of the formed product cone, applying equation (1).

$$
\alpha^{\circ}=\arctan *\left(\frac{2 h}{d}\right)
$$

where $\mathrm{h}=$ height from the top of the formed product cone $(\mathrm{cm})$; $\mathrm{d}=$ maximum cone product diameter $(\mathrm{cm})$, taken as an average of at least 6 values.

\subsection{Wettability}

A modified UNE ${ }^{[2]}$ standard method for milk powder was used. Briefly $10 \mathrm{~g}$ of powder were weighed and dropped progressively (in $25 \mathrm{~s}$ ) into $250 \mathrm{~g}$ of water, measuring the time (s) from when the powder falls until all the particles in the sample have been wetted.

\subsection{Bulk flow properties}

The porosity $(\varepsilon)$ was calculated from the true and bulk densities by using equation (2). The true density $(\rho)$ of the sample was calculated from its individual components. In this case, water and carbohydrates, both own and added, were considered to be the main components of the samples (equation 3 ). The apparent density $\left(\rho_{a}\right)$ was calculated by dropping the sample in a graduated tube until approximately $10 \mathrm{~mL}$, with the help of a funnel; the weight and volume of the exact sample was recorded. Finally the previous sample was compacted using a vortex (1200 rpm, t = $10 \mathrm{~s}$ ) and the volume of the sample was recorded again to calculate the value of bulk density $\left(\rho_{b}\right)$. Based on these data it was possible to calculate the Hausner Index and the Carr Index by using equations (4) and (5).

$$
\begin{gathered}
\varepsilon=\frac{\rho-\rho_{b}}{\rho} \\
\frac{1}{\rho}=\frac{x_{w}}{\rho_{w}}+\frac{x_{C H}}{\rho_{C H}} \\
I_{H}=\frac{\rho_{b}}{\rho_{a}} \\
I_{C}=\frac{\rho_{b}-\rho_{a}}{\rho_{b}}
\end{gathered}
$$

where $\varepsilon$ is the porosity; $\rho, \rho_{b}$ and $\rho_{a}(\mathrm{~g} / \mathrm{cc})$ are the true, bulk and apparent densities of the powder, respectively; $\mathrm{x}(\mathrm{w} / \mathrm{w}), \rho_{\mathrm{w}}(\mathrm{g} / \mathrm{cc})$ and $\rho_{\mathrm{cH}}(\mathrm{g} / \mathrm{cc})$ are the mass fraction and density (20 ${ }^{\circ} \mathrm{C}$ ) of water $\left(0.9976 \mathrm{~g} / \mathrm{cc}^{[3]}\right)$ and carbohydrates $\left(1.4246 \mathrm{~g} / \mathrm{Cc}^{[3]}\right)$, respectively. 


\section{Results and Discussion}

The particle size distribution is represented in figure 1. All the samples had the greater proportion of particles with a size lesser than $100 \mu \mathrm{m}$. In these range, OD sample stands out above the other two samples.

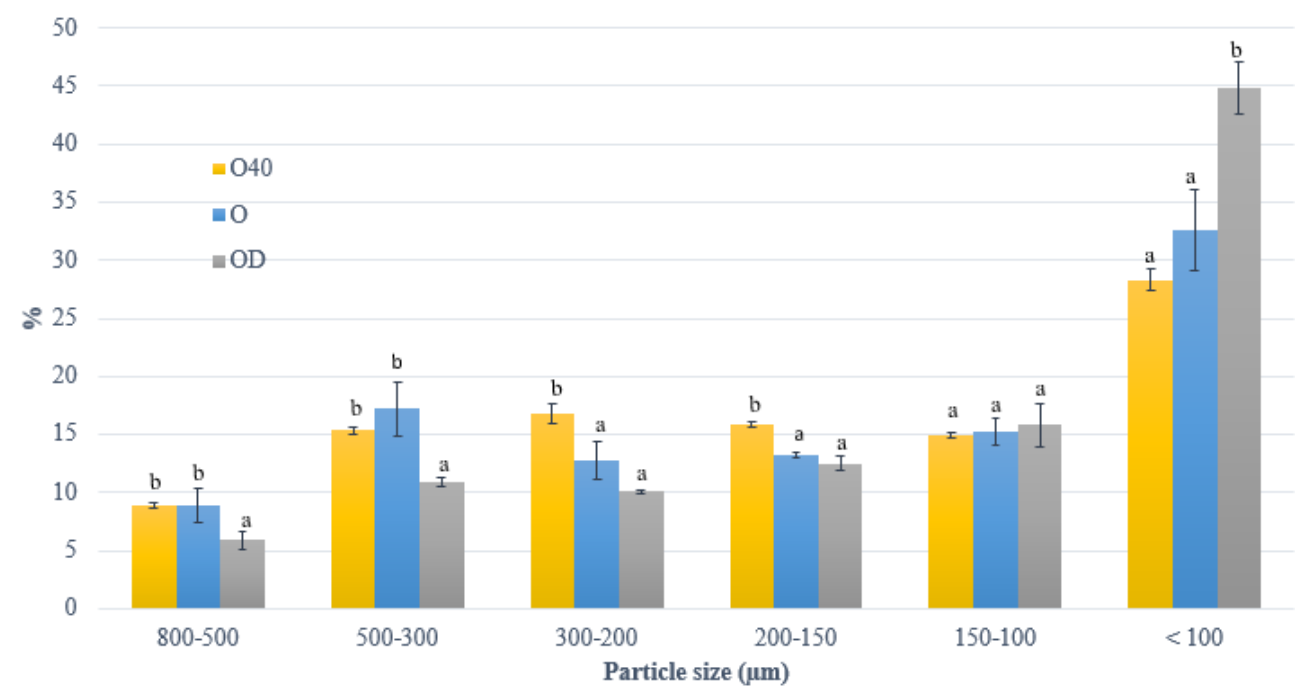

Figure 1. Relative powder particle size distribution ( $\mu \mathrm{m})$ obtained from the formulated puree orange freeze-dried at room temperature $(O)$, formulated and pre-dehydrated puree orange freezedried at room temperature (OD) and formulated puree orange freeze-dried at $40{ }^{\circ} \mathrm{C}(\mathrm{O} 40)$.

The pondered average size particle of each sample was calculated from powder particle size distribution (table 1). Significant differences $(\mathrm{p}<0.05)$ were observed among all the samples, sample OD40 being the one with the greatest average particle size and sample OD the one with the smallest average particle size. Particle size may be related with the fracture resistance of the cake when crushed, the greater the resistance the greater the particle size. The increase in the solutes concentration of the microwave pre-dehydrated sample will lead to a decrease in the freezing rate. In this case, greater ice crystals are expected to be formed. This will suppose to obtain a cake with greater porous size and more fragile. On the other hand, when $40^{\circ} \mathrm{C}$ shelf temperature is applied during freeze-drying, the drying of the sample is accelerated. In this case, smaller pores size are formed and a more resistant cake is obtained (data not publicated).

Measured properties related to powders flowability are presented in table 1. Samples doesn't show sifnificant differences $(\mathrm{p}>0.05)$ regarding the angle of repose. The values ranged from $40,35^{\circ}$ to $42,51^{\circ}$, which are included among acceptable flow properties ${ }^{[4]}$. 
Table 1. Mean values ( \pm standard deviation) of the properties measured to the powder obtained from the formulated puree orange freeze-dried at room temperature $(O)$, formulated and predehydrated puree orange freeze-dried at room temperature $(O D)$ and formulated puree orange freeze-dried at $40^{\circ} \mathrm{C}(040)$.

\begin{tabular}{|c|c|c|c|}
\hline \multirow[b]{2}{*}{ Property } & \multicolumn{3}{|c|}{ Sample } \\
\hline & $\mathbf{O 4 0}$ & $\mathbf{O}$ & OD \\
\hline Average size particle (mm) & $0.210^{\mathrm{a}} \pm 0.008$ & $0.1703^{b} \pm 0.0006$ & $0.117^{c} \pm 0.004$ \\
\hline Angle of repose $\left(^{\circ}\right)$ & $43^{\mathrm{a}} \pm 2$ & $40.3^{\mathrm{a}} \pm 0.5$ & $41.7^{\mathrm{a}} \pm 0.5$ \\
\hline Wettabillity (s) & $571^{b} \pm 54$ & $467^{b} \pm 18$ & $1924^{\mathrm{a}} \pm 400$ \\
\hline Porosity (\%) & $77.1^{\mathrm{a}} \pm 0.6$ & $80.1^{\mathrm{a}} \pm 1.4$ & $69^{b} \pm 3$ \\
\hline Hausner index & $1.124^{\mathrm{a}} \pm 0.018$ & $1.11^{\mathrm{a}} \pm 0.06$ & $1.20^{\mathrm{a}} \pm 0.08$ \\
\hline Carr index (\%) & $11.0^{\mathrm{a}} \pm 1.4$ & $10^{\mathrm{a}} \pm 5$ & $17^{\mathrm{a}} \pm 6$ \\
\hline
\end{tabular}

The same lowercase letter within rows indicates homogeneous groups established by ANOVA $(p<0.05)$.

The wetting time is inversed related to wettability, the higher the wetting time the worse the wettability. In this way, the microwave pre-dehydrated sample before freeze-drying (OD, table 1) showed the worst degree of wetting. This probably may be related with the smaller average particle size of this sample $\mathrm{e}^{[5]}$. The lesser particle size induces the particles aggregation and the lump formation.

It is well recognized that dehydrated products obtained from freeze-drying are highly porous $^{[6]}$. However, it is observed that OD sample, which was microwave pre-dehydrated before freeze-drying, shows a value of porosity much lower than the other two samples (table 1). This may also be related to the smaller average particle size of this sample. It has been observed that porosity decreased with decreasing the particle size, because of the decrease in the inter-particle voids with smaller sized particles ${ }^{[6]}$.

Hausner and Carr indexes indicate the level of interaction between particles and therefore the flow properties. There was no significant differences $(\mathrm{p}<0.05)$ between the values of both indexes for the samples. According the classification of Farmacopea ${ }^{[4]}$, all the samples show an excellent to acceptable flowability.

\section{Conclusions}

The different freeze-drying conditions used in this study doesn't affect the flowability of the freeze-dried orange powder. Nevertheless, pre-dehydration of the formulated orange puree decreases the mechanical resistance of the obtained cake so that a powder with a smaller average particle size may be obtained. This affects the rehydration capacity of the freezedried powder, with increased wetting time. In this sense, $40^{\circ} \mathrm{C}$ shelf temperature during 
freeze-drying may be recommended in order to short the process time and to get a product with both a good flow behavior and good wettability.

\section{Acknowledgements}

The authors thank the Ministerio de Economía, Industria y Competitividad for the financial support given through the Project AGL 2017-89251-R (AEI/FEDER-UE) and the Ministerio of Educación, Cultura y Deporte for the FPU grant (FPU14 / 02633) granted to Ms. Andrea Silva.

\section{References}

[1] Martínez-Navarrete, N.; Camacho, M.M.; Martínez-Lahuerta, J.J. Los compuestos bioactivos de las frutas y sus efectos sobre la salud, 2008, Actividad Dietética, 12(2), 64-68.

[2] UNE 34849, Instant dried milk. Determination of dispersibility and wettability, 1986.

[3] Choi, Y.; Okos, M.R. Thermal properties of liquid foods. Review. In: Physical and Chemical properties of food, M.R. Okos (Ed.), American Society of Agricultural Engineers, Michigan, USA, 1986, pp. 35-77.

[4] Real Farmacopea Española. Ministerio de Sanidad, Servicios Sociales e Igualdad. Agencia Española de Medicamentos y Productos Sanitarios. fifth ed. Madrid, 2015.

[5] Ferrari, C.C.; Marconi, S.P.; Dutra, I.; Zaratini, F.; de Aguirre, J.M. Influence of carrier agents on the physicochemical properties of blackberry powder produced by spray drying. International Journal of Food Science and Technology, 2012, 47(6), 1237-1245.

[6] Caparino, O. A.; Tang, J.; Nindo, C.I.; Sablani, S.S.; Powers, J.R.; Fellman, J.K. Effect of drying methods on the physical properties and microstructures of mango (Philippine “Carabao” var.) powder. Journal of Food Engineering, 2012, 111(1), 135-148. 